\title{
Exigências térmicas, estimativa do número de gerações de Stenoma catenifer e comprovação do modelo em campo
}

\author{
Dori Edson $\operatorname{Nava}^{(1)}$, Marinéia de Lara Haddad(1) e José Roberto Postali Parra(1)
}

(1)Universidade de São Paulo, Escola Superior de Agricultura Luiz de Queiroz, Dep. de Entomologia, Fitopatologia e Zoologia Agrícola, Caixa Postal 9, CEP 13418-90 Piracicaba, SP. E-mail: nava@esalq.usp.br, mlhaddad@esalq.usp.br, jrpparra@esalq.usp.br

\begin{abstract}
Resumo - O objetivo deste trabalho foi estudar a biologia de Stenoma catenifer em diferentes temperaturas, determinar suas exigências térmicas, estimar o número de gerações para o Município de São Tomás de Aquino, MG e comprovar o modelo em campo. Para a determinação da duração, viabilidade, fecundidade, longevidade e exigências térmicas, criaram-se insetos em sementes de abacate cultivar Breda, em diferentes temperaturas, umidade relativa de $70 \pm 10 \%$ e fotófase de 14 horas. A duração das fases de desenvolvimento e do ciclo biológico (ovo-adulto) foi afetada pela temperatura, tendo sido maior nas temperaturas mais baixas; a viabilidade foi maior na faixa térmica de $18^{\circ} \mathrm{C}$ a $28^{\circ} \mathrm{C}$. O limiar térmico inferior de desenvolvimento $(\mathrm{Tb})$ e a constante térmica $(\mathrm{K})$ para as fases de ovo, lagarta, pupa e período ovo-adulto foram de $9,1^{\circ} \mathrm{C}$ e $82,3 \mathrm{GD} ; 8,3^{\circ} \mathrm{C}$ e $398,4 \mathrm{GD} ; 10,1^{\circ} \mathrm{C}$ e $164,7 \mathrm{GD}$ e $8,9^{\circ} \mathrm{C}$ e $644,5 \mathrm{GD}$, respectivamente. A temperatura influenciou a capacidade de postura e a longevidade de machos e fêmeas. Com base nas normais térmicas, os números de gerações anuais e por ciclo de produção de abacate foram de 7,8 e 5,1, respectivamente. A flutuação populacional de S. catenifer pode ser utilizada como um indicativo do número de gerações, baseada nas exigências térmicas da praga.
\end{abstract}

Termos para indexação: Insecta, Lepidoptera, Elachistidae, broca-do-abacate, abacate.

\section{Temperature requirements, estimate of the generations number of Stenoma catenifer and verification of the model in the field}

\begin{abstract}
The objective of this work was to study the biology of Stenoma catenifer under different temperatures, determine its temperature requirements, estimate the number of generations for the city of São Tomás de Aquino, MG, and verify the model in the field. In order to determine duration, viability, fecundity, longevity, and temperature requirements, the insects were reared on cultivar Breda avocado seeds at different temperatures, under $70 \pm 10 \%$ relative humidity and a 14-hour photophase. Developmental stages and biological cycle (egg-adult) durations were affected by temperature, with higher values at lower temperatures, while viability was highest at temperature ranging from $18^{\circ} \mathrm{C}$ to $28^{\circ} \mathrm{C}$. The lowest temperature development threshold (Tb), and thermal constant (K) for the egg, caterpillar, and pupal stages, and for the egg-adult period were $9.1^{\circ} \mathrm{C}$ and $82.3 \mathrm{GD}, 8.3^{\circ} \mathrm{C}$ and $398.4 \mathrm{GD}, 10.1^{\circ} \mathrm{C}$ and 164.7 GD, and $8.9^{\circ} \mathrm{C}$ and $644.5 \mathrm{GD}$, respectively. Temperature influenced the egg-laying capacity and longevity of females and males. Based on the normal values of temperature, the numbers of generations per year and per production cycle were 7.8 and 5.1, respectively. The population fluctuation of S. catenifer can be used as an indicator of the number of generations, based on temperature requirements of the pest.
\end{abstract}

Index terms: Insecta, Lepidoptera, Elachistidae, avocado fruit borer, avocado.

\section{Introdução}

Stenoma catenifer Walsingham, 1912 (Lepidoptera: Elachistidae), popularmente conhecida como broca-do-abacate, é uma espécie neotropical relatada na Venezuela (Boscán de Martínez \& Godoy, 1984), Peru (Arellano Cruz, 2001) e México (Acevedo et al., 1972). No Brasil, Costa Lima (1945) se referiu à presença desse inseto em 1923 e, desde então, vem sendo relatado como praga do abacateiro; tem distribuição generalizada e é freqüente nos principais estados produtores (SP, MG, ES e PR). As mariposas realizam posturas em fissuras, pedicelos e pedúnculos dos frutos. As lagartas eclodem e perfuram a casca (exocarpo), alimentam-se inicialmente da polpa (endocarpo), até atingir o caroço, e causam sérios danos aos frutos, com perdas de até $100 \%$ da produção (Hohmann \& Meneguim, 1993). 
Esse inseto é, atualmente, considerado a principal praga do abacate, provoca graves prejuízos econômicos e, em muitos casos, abandono das áreas de produção pelo agricultor, em conseqüencia da falta de um conjunto de medidas que sejam eficazes e causem pouco impacto ambiental. O principal método de controle empregado é o químico, e os inseticidas do grupo químico dos piretróides são os mais utilizados (Hohmann et al., 2000). A falta de tecnologias de aplicação para a cultura e o desconhecimento da bioecologia da praga têm sido fatores determinantes para o insucesso do controle químico. O controle biológico aplicado não é utilizado, por falta de técnicas de criação e de liberação, já que vários inimigos naturais da broca-do-abacate podem ocorrer no campo, destacando-se os parasitóides de ovos da família Tricogrammatidae, com registros de parasitismo de até 40\% (Hohmann \& Meneguim, 1993), e parasitóides larvais das famílias Braconidae e Ichneumonidae, com parasitismos que variam em 9\% (Hohmann \& Meneguim, 1993) a 30\% (Boscán de Martínez \& Godoy, 1982). O controle cultural, através da catação dos frutos atacados, na planta e no chão, também auxilia no manejo da praga, porém é uma medida de alto custo.

Muitas vezes, o insucesso no controle se deve à época inadequada de aplicação dos diferentes métodos utilizados. A previsão de ocorrência da praga, com base nas exigências térmicas, pode melhorar a eficiência de controle. A utilização de modelos de graus-dia de laboratório tem auxiliado os programas de manejo (Higley et al., 1986; Ávila et al., 2002; Nava \& Parra, 2003), e tem permitido conhecer a dinâmica populacional de pragas, determinar com mais eficiência a época de amostragem e definir o número de gerações delas.

O objetivo desta pesquisa foi estudar a biologia de $S$. catenifer em diferentes temperaturas, determinar as exigências térmicas das diferentes fases de desenvolvimento, estimar o número de gerações anuais e durante o ciclo de produção (dezembro-agosto), para a região de São Tomás de Aquino, MG, com base em suas normais térmicas, além de buscar sua comprovação, tendo por base a dinâmica populacional da praga, em condições de campo.

\section{Material e Métodos}

Os insetos utilizados nos experimentos foram criados no Laboratório de Biologia de Insetos, do Departamento de Entomologia, Fitopatologia e Zoologia Agrícola, da Escola Superior de Agricultura Luiz de Queiroz (Esalq), Universidade de São Paulo (USP), em Piracicaba, SP, segundo metodologia proposta por Nava (2005). Para estabelecimento da criação de manutenção, coletaram-se insetos num pomar de abacate, cultivares Breda e Margarida, da Empresa Café Total, no Município de São Tomás de Aquino, MG (2052'30" S, 4707'30" W e 1.000 m de altitude).

Para o estudo das exigências térmicas, do período embrionário, foram utilizados ovos de Stenoma catenifer da criação de manutenção. O papel que serviu de substrato de postura foi recortado com 25 ovos e transferido para placas de Petri com papel de filtro, no fundo, tratado com $\mathrm{CuSO}_{4}$ a $1 \%$, por 5 minutos, para evitar contaminações fúngicas. As placas de Petri foram fechadas e vedadas com filme de plástico PVC, e levadas a sete câmaras climatizadas, reguladas a $18,20,22,25,28,30$ e $32^{\circ} \mathrm{C}$, com umidade relativa de $70 \pm 10 \%$ e fotófase de 14 horas, tendo sido verificada, diariamente, a eclosão de lagartas.

Para a determinação das exigências térmicas da fase larval, os insetos foram criados em sementes de abacate da cultivar Breda. As lagartas foram individualizadas em recipientes de acrílico transparente $(10 \mathrm{~cm}$ de altura e $10 \mathrm{~cm}$ e $8 \mathrm{~cm}$ de base e topo, respectivamente), com papel filtro em seu interior, para reter a umidade e, sobre este, colocou-se uma semente de abacate. As lagartas foram criadas nas temperaturas anteriormente citadas até a fase de pupa, avaliando-se a duração e a viabilidade.

Para o desenvolvimento pupal, foram utilizados insetos provenientes da criação de manutenção, as pupas foram individualizadas em placas de acrílico $(6 \mathrm{~cm}$ de diâmetro x 1,5 cm de altura), com algodão embebido em água, para a manutenção da umidade. Diariamente, a duração e a viabilidade da fase de pupa foram avaliadas.

O delineamento experimental foi inteiramente casualizado, com sete tratamentos (temperatura) e seis repetições, cada uma delas constituída de 25 ovos, 25 lagartas e 25 pupas, num total de 150 insetos por tratamento. A partir dos resultados obtidos, determinou-se o limite térmico inferior de desenvolvimento ( $\mathrm{Tb})$ e a constante térmica (K), pelo método da hipérbole, utilizandose o software MOBAE (Modelos Bioestatísticos Aplicados à Entomologia) (Haddad et al., 1999). Os dados de duração e viabilidade foram submetidos à análise de variância ANOVA, e as médias comparadas pelo teste de Tukey $(\mathrm{p}<0,05)$.

O número provável de gerações anuais e por ciclo de produção (dezembro-agosto) de $S$. catenifer foi estimado segundo Parra (1985), utilizando-se o número de graus-dia necessários para o desenvolvimento do inse- 
to, determinado em laboratório, e as normais térmicas (1986-2002) do Município de São Tomás de Aquino, MG, obtidas na estação meteorológica da Empresa Agropecuária de Minas Gerais (Epamig). O número de gerações estimado, a partir do modelo de exigências térmicas determinado em laboratório, foi confrontado com a flutuação populacional da broca-do-abacate, durante as safras agrícolas de 2001/2002 e 2002/2003, para comprovação da eficiência do modelo. A duração de cada geração foi obtida por meio do somatório diário dos graus-dia, determinado em laboratório para o período de ovo-adulto, tendo como início o mês de dezembro.

Para o estudo da flutuação populacional, foi realizado um levantamento no Município de São Tomás de Aquino, MG, em pomares de abacate da cultivar Breda, de aproximadamente 3 ha, duas vezes por mês, durante o período de dezembro a agosto. A amostragem foi realizada de forma seqüencial em 11 plantas; foram coletados 4 frutos na parte mediana, num total de 44 frutos, que foram transportados para o laboratório e examinados, e foi verificada a presença de lagartas.

Para a determinação da longevidade e da fecundidade, foram individualizados 25 casais em gaiolas de PVC, de $23 \mathrm{~cm}$ de altura por $15 \mathrm{~cm}$ de diâmetro, conforme descrito para a criação de manutenção, mantidas em temperatura constante de $20^{\circ} \mathrm{C}, 25^{\circ} \mathrm{C}$ e $30^{\circ} \mathrm{C}$, umidade relativa de $70 \pm 10 \%$ e fotófase de 14 horas. Essas temperaturas foram selecionadas por representarem a faixa térmica das regiões em estudo. $\mathrm{O}$ alimento foi trocado diariamente tendo-se registrado o número de ovos e a mortalidade. O delineamento experimental foi inteira- mente casualizado, com três tratamentos (temperaturas) e 25 repetições (casais). Os dados de fecundidade e longevidade de machos e fêmeas foram submetidos à análise da variância, e as médias foram comparadas pelo teste de Tukey $(\mathrm{p}<0,05)$.

\section{Resultados e Discussão}

A duração e a viabilidade das diferentes fases do ciclo biológico de Stenoma catenifer foram variáveis em função da temperatura (Tabelas 1 e 2). No período embrionário houve uma diminuição da duração, com o aumento da temperatura, na faixa térmica de $18^{\circ} \mathrm{C}$ (9,9 dias) a $30^{\circ} \mathrm{C}\left(4,1\right.$ dias); a $32^{\circ} \mathrm{C}$ o período de incubação não se alterou, tendo mostrado uma tendência de inadequação à fase de ovo, nas temperaturas superiores a $30^{\circ} \mathrm{C}$, embora esse valor tenha sido estatisticamente semelhante a $28^{\circ} \mathrm{C}, 30^{\circ} \mathrm{C}$ e $32^{\circ} \mathrm{C}$. Na temperatura de $25^{\circ} \mathrm{C}$, a duração foi de 5 dias, próxima àquela relatada por Jaramillo et al. (1972) de 5 a 6 dias, e por Hohmann \& Meneguim (1993) de 6 dias. A viabilidade foi elevada em todas as temperaturas, exceto na de $32^{\circ} \mathrm{C}$, cujo valor foi bem aquém daquele registrado nas demais temperaturas (Tabela 2).

Para a fase larval, a tendência do efeito negativo de altas temperaturas se manteve, pois não ocorreu desenvolvimento larval a $32^{\circ} \mathrm{C}$, e não ocorreram diferenças na duração da fase larval a $28^{\circ} \mathrm{C}$ e $30^{\circ} \mathrm{C}$ (Tabela 1). Esse efeito negativo das altas temperaturas, sobre o desenvolvimento larval, pôde ser evidenciado na viabilidade, já que a $30^{\circ} \mathrm{C}, 43 \%$ dos insetos morreram.

Tabela 1. Duração média (D) ( \pm erro-padrão da média) e intervalo de variação (IV), em dias, das fases de ovo, larval, pupal e do ciclo biológico (ovo-adulto) de Stenoma catenifer, em sementes de abacate cultivar Breda, em diferentes temperaturas, sob umidade relativa de $70 \pm 10 \%$ e fotófase de 14 horas $^{(1)}$.

\begin{tabular}{|c|c|c|c|c|c|c|c|c|}
\hline \multirow{2}{*}{$\begin{array}{l}\text { Temperatura } \\
\left({ }^{\circ} \mathrm{C}\right)\end{array}$} & \multicolumn{2}{|c|}{ Fase de ovo } & \multicolumn{2}{|c|}{ Fase larval } & \multicolumn{2}{|c|}{ Fase pupal } & \multicolumn{2}{|c|}{ Ciclo biológico } \\
\hline & $\mathrm{D}$ & IV & $\mathrm{D}$ & IV & $\mathrm{D}$ & IV & $\mathrm{D}$ & IV \\
\hline 18 & $9,9 \pm 0,07 a$ & $10,1-9,7$ & $39,4 \pm 0,24 a$ & $38,7-40,2$ & $20,2 \pm 0,64 a$ & $19,4-20,8$ & $69,5 \pm 0,57 \mathrm{a}$ & $67,9-71,6$ \\
\hline 20 & $7,5 \pm 0,11 b$ & $7,8-7,2$ & $36,8 \pm 0,12 b$ & $36,4-37,1$ & $16,9 \pm 0,58 \mathrm{~b}$ & $16,3-17,7$ & $61,2 \pm 0,36 \mathrm{~b}$ & $60,8-62,4$ \\
\hline 22 & $6,3 \pm 0,05 c$ & $6,4-6,2$ & $27,9 \pm 0,23 \mathrm{c}$ & $27,4-29,0$ & $14,1 \pm 0,18 \mathrm{c}$ & $13,9-14,4$ & $47,8 \pm 0,25 \mathrm{c}$ & $47,3-49,2$ \\
\hline 25 & $5,0 \pm 0,02 d$ & $5,1-4,9$ & $24,3 \pm 0,34 d$ & $23,2-25,6$ & $11,0 \pm 0,23 \mathrm{~d}$ & $10,7-11,4$ & $40,3 \pm 0,33 d$ & $39,4-41,7$ \\
\hline 28 & $4,3 \pm 0,17 \mathrm{e}$ & $4,6-6,7$ & $19,1 \pm 0,90 \mathrm{e}$ & $17,8-23,7$ & $8,9 \pm 0,08 \mathrm{e}$ & $8,8-9,0$ & $32,4 \pm 0,84 \mathrm{e}$ & $30,8-36,2$ \\
\hline 30 & $4,1 \pm 0,03 \mathrm{e}$ & $4,1-3,9$ & $18,9 \pm 0,15 f$ & $18,3-19,2$ & $8,4 \pm 0,25 \mathrm{e}$ & $8,1-8,8$ & $31,4 \pm 0,11 \mathrm{e}$ & $30,8-31,6$ \\
\hline 32 & $4,2 \pm 0,02 \mathrm{e}$ & $4,3-4,2$ & \multicolumn{2}{|c|}{-} & \multicolumn{2}{|c|}{-} & \multicolumn{2}{|c|}{-} \\
\hline $\mathrm{F}$ & \multicolumn{2}{|c|}{213,0544} & 419 , & & \multicolumn{2}{|c|}{$\begin{array}{c}857,9685 \\
000001\end{array}$} & \multicolumn{2}{|c|}{758,0715} \\
\hline $\mathrm{P}$ & \multicolumn{2}{|c|}{0,00001} & \multicolumn{2}{|c|}{0,00001} & 0,0 & & \multicolumn{2}{|c|}{0,00001} \\
\hline $\mathrm{df}$ & \multicolumn{2}{|c|}{5} & \multicolumn{2}{|c|}{5} & \multicolumn{2}{|c|}{5} & \multirow{2}{*}{\multicolumn{2}{|c|}{$\begin{array}{c}5 \\
450 \\
\end{array}$}} \\
\hline $\mathrm{n}$ & \multicolumn{2}{|c|}{150} & \multicolumn{2}{|c|}{150} & \multicolumn{2}{|c|}{150} & & \\
\hline
\end{tabular}

(1)Médias seguidas da mesma letra, nas colunas, não diferem estatisticamente entre si, pelo teste de Tukey a 5\% de probabilidade. 
Na temperatura de $25^{\circ} \mathrm{C}$, a duração da fase larval de S. catenifer foi de 24,3 dias, valor superior aos 15,3 dias encontrados por Hohmann \& Meneguim (1993), e aos 19,8 dias observados por Bóscan de Martínez \& Godoy (1984), que criaram o inseto com polpa de abacate, cultivares Margarida e Ceniap, respectivamente.

Cervantes et al. (1999) relataram uma duração de 20 dias para insetos criados em "Greenheart" Chlorocardium rodiei (Schomb.) Rohwer Richter \& van der Werff. Essas diferenças podem estar relacionadas com o substrato alimentar, uma vez que, no presente trabalho, utilizou-se semente de abacate cultivar Breda, enquanto os autores citados criaram S. catenifer em polpa de abacate de diferentes cultivares ou sementes de outra espécie (C. rodiei).

A viabilidade também foi afetada pelas diferentes temperaturas, tendo sido menor na temperatura de $30^{\circ} \mathrm{C}$ $(57,8 \%)$ e diferido das demais, que foram superiores a $72 \%$ (Tabela 2). Esse alto porcentual de viabilidade contrasta com o valor de 25,4\%, obtido por Hohmann \& Meneguim (1993) (Tabela 1). Isto pode ser explicado pelo fato de aqueles autores terem utilizado frutos para o desenvolvimento larval, enquanto, no presente trabalho, o inseto foi criado em sementes, que são mais resistentes ao ataque dos microrganismos e, aparentemente, conservam a qualidade nutricional do alimento.

De maneira semelhante ao ocorrido na fase larval, as altas temperaturas foram prejudiciais para o desenvolvimento da fase pupal, de modo que a duração a $30^{\circ} \mathrm{C}$ (8,4 dias) não diferiu, significativamente, da de $28^{\circ} \mathrm{C}$ (8,9 dias) (Tabela 1 ). Na temperatura de $25^{\circ} \mathrm{C}$, a dura- ção foi de 11 dias, próxima dos 10,6 e 12,1 dias relatados por Hohmann \& Meneguim (1993) e Cervantes et al. (1999), respectivamente. A viabilidade da fase pupal foi superior a $80 \%$ em todas as temperaturas (Tabela 2), com exceção de $30^{\circ} \mathrm{C}$, que foi de $60,8 \%$. A alta viabilidade dessa fase foi também verificada por Hohmann \& Meneguim (1993).

A duração total do ciclo biológico (ovo-adulto) aumentou com a diminuição da temperatura, tendo variado de 69,5 dias a $18^{\circ} \mathrm{C}$ a 31,4 dias a $30^{\circ} \mathrm{C}$ (Tabela 1 ). Apesar da duração na temperatura de $30^{\circ} \mathrm{C}$ (31,4 dias) ser menor do que a de $28^{\circ} \mathrm{C}$ (32,3 dias), não se observaram diferenças significativas entre tais durações. A viabilidade total foi superior a 52,3\%, com exceção da temperatura de $30^{\circ} \mathrm{C}$, que foi de $28,7 \%$ (Tabela 2). Assim, conclui-se que temperaturas altas (superiores a $30^{\circ} \mathrm{C}$ ) são prejudiciais ao desenvolvimento de $S$. catenifer, uma vez que a viabilidade nas diferentes fases do desenvolvimento, com exceção da de ovo, sempre foi inferior, tendo diferido, significativamente, das demais temperaturas estudadas. Já na menor temperatura $\left(18^{\circ} \mathrm{C}\right)$, apesar de haver um alongamento do ciclo biológico, observou-se que a viabilidade foi alta, o que indica que, para essa espécie, as baixas temperaturas são favoráveis ao desenvolvimento.

Para as fases de ovo, larva, pupa e ciclo biológico (ovo-adulto), determinou-se um limiar térmico inferior de desenvolvimento (temperatura base) de $9,1^{\circ} \mathrm{C} ; 8,3^{\circ} \mathrm{C}$; $10,1^{\circ} \mathrm{C}$ e $8,9^{\circ} \mathrm{C}$, respectivamente, com constantes térmicas de 82,3, 398,4, 164,7 e 644,5 GD, respectivamente (Tabela 3). O coeficiente de determinação foi superior

Tabela 2. Viabilidade média (V) ( \pm erro-padrão da média) e intervalo de variação (IV), em dias, das fases de ovo, larval, pupal e do ciclo biológico (ovo-adulto) de Stenoma catenifer, em sementes de abacate cultivar Breda, em diferentes temperaturas, sob umidade relativa de $70 \pm 10 \%$ e fotófase de 14 horas $^{(1)}$.

\begin{tabular}{|c|c|c|c|c|c|c|c|c|}
\hline \multirow{2}{*}{$\begin{array}{c}\text { Temperatura } \\
\left({ }^{\circ} \mathrm{C}\right)\end{array}$} & \multicolumn{2}{|c|}{ Fase de ovo } & \multicolumn{2}{|c|}{ Fase larval } & \multicolumn{2}{|c|}{ Fase pupal } & \multicolumn{2}{|c|}{ Ciclo biológico } \\
\hline & $\mathrm{V}$ & IV & $\mathrm{V}$ & IV & $\mathrm{V}$ & IV & $\mathrm{V}$ & IV \\
\hline 18 & $90,8 \pm 3,0 \mathrm{a}$ & $98,6-83,1$ & $85,6 \pm 3,2 \mathrm{a}$ & $80,0-100,0$ & $84,2 \pm 10,2 a$ & $70,0-95,0$ & $65,4 \pm 4,7 \mathrm{a}$ & $50,4-82,3$ \\
\hline 20 & $90,8 \pm 3,0 \mathrm{a}$ & $98,6-83,1$ & $81,1 \pm 3,2 \mathrm{a}$ & $73,3-93,3$ & $87,5 \pm 9,9 a$ & $75,0-95,0$ & $64,5 \pm 3,1 \mathrm{a}$ & $49,5-69,9$ \\
\hline 22 & $90,8 \pm 3,0 \mathrm{a}$ & $98,6-83,1$ & $78,9 \pm 4,0 \mathrm{a}$ & $66,7-93,3$ & $88,3 \pm 10,3 a$ & $70,0-100,0$ & $63,2 \pm 5,6 a$ & $43,9-79,8$ \\
\hline 25 & $91,7 \pm 3,3 \mathrm{a}$ & $100,0-83,1$ & $79,9 \pm 6,2 \mathrm{a}$ & $53,3-93,3$ & $87,5 \pm 6,1 \mathrm{a}$ & $80,0-95,0$ & $64,2 \pm 3,5 \mathrm{a}$ & $50,6-75,4$ \\
\hline 28 & $81,2 \pm 4,1 \mathrm{a}$ & $95,3-68,1$ & $72,2 \pm 4,4 a$ & $53,3-86,7$ & $89,2 \pm 7,4 a$ & $80,0-100,0$ & $52,3 \pm 2,8 \mathrm{a}$ & $43,1-62,7$ \\
\hline 30 & $81,7 \pm 5,3 \mathrm{a}$ & $91,8-70,5$ & $57,8 \pm 7,8 b$ & $33,0-86,7$ & $60,8 \pm 21,3 b$ & $30,0-85,0$ & $28,7 \pm 2,9 b$ & $14,4-33,0$ \\
\hline 32 & $62,3 \pm 2,8 \mathrm{~b}$ & $69,4-55,3$ & \multicolumn{2}{|l|}{-} & \multicolumn{2}{|c|}{-} & \multicolumn{2}{|c|}{-} \\
\hline $\mathrm{F}$ & \multicolumn{2}{|c|}{6,8206} & \multicolumn{2}{|c|}{3,7764} & \multicolumn{2}{|c|}{5,0546} & \multicolumn{2}{|c|}{15,2242} \\
\hline$P$ & \multicolumn{2}{|c|}{0,00017} & \multicolumn{2}{|c|}{0,00913} & \multicolumn{2}{|c|}{0,00207} & \multicolumn{2}{|c|}{0,00001} \\
\hline $\mathrm{df}$ & \multicolumn{2}{|c|}{5} & \multicolumn{2}{|c|}{5} & \multicolumn{2}{|c|}{5} & \multicolumn{2}{|c|}{5} \\
\hline $\mathrm{n}$ & \multicolumn{2}{|c|}{150} & \multicolumn{2}{|c|}{150} & \multicolumn{2}{|c|}{150} & \multicolumn{2}{|c|}{450} \\
\hline
\end{tabular}

(1)Médias seguidas da mesma letra, nas colunas, não diferem estatisticamente entre si, pelo teste de Tukey a 5\% de probabilidade. 
a 0,974, acima do preconizado pelo método da hipérbole $(0,900)$. Verificou-se que o limite térmico inferior de desenvolvimento da fase de pupa diferiu, estatisticamente, a 5\% de probabilidade, do limite térmico inferior da fase de lagarta e do período ovo-adulto, pois seus intervalos de confiança não se sobrepuseram (Tabela 3).

Segundo Honék (1996), o limiar térmico inferior de desenvolvimento diminui com o aumento da latitude. Espécies que vivem nos trópicos, possuem uma temperatura base maior $\left(13,7^{\circ} \mathrm{C}\right)$ do que espécies que vivem em regiões subtropicais $\left(10,5^{\circ} \mathrm{C}\right)$ e regiões temperadas $\left(7,9^{\circ} \mathrm{C}\right)$. No entanto, o valor do limiar térmico inferior, do ciclo biológico (ovo-adulto) de S. catenifer $\left(8,9^{\circ} \mathrm{C}\right.$ ), faz com que tal regra não seja aplicável para essa espécie neotropical. Fatores como a altitude também deveriam ser levados em conta para a classificação dos insetos, quanto às exigências térmicas, já que a temperatura diminui com o aumento da altitude. Além disso, sabe-se que a alimentação (Zeiss et al., 1996; Mihsfeldt, 1998) e a origem geográfica (diferentes populações) (Bleicher \& Parra, 1990) também influenciam na determinação das exigências térmicas.

A quantidade de graus-dia (GD) acumulados acima do limiar térmico inferior de desenvolvimento $\left(8,89^{\circ} \mathrm{C}\right)$, anualmente e por ciclo de produção (dezembro-agosto), foi de 4.996,8 e 3.302,0 GD, perfazendo 7,8 e 5,1 gerações, respectivamente. A partir da análise da flutuação populacional de S. catenifer, na safra agrícola 2001/ 2002, foram observados 6 picos populacionais, correspondentes às prováveis gerações (Figura 1), com base nos dados de laboratório. Para a safra agrícola 2002/ 2003, observou-se que a flutuação populacional apresentou um pico a menos, em relação à safra anterior, ou seja, 5 gerações (Figura 1), próximo ao número determinado em laboratório, que foi de 5,1 gerações.

Durante os meses mais frios (maio, junho, julho e agosto), deve ter havido um alongamento do período de desenvolvimento de $S$. catenifer, o que levou a uma maior distância entre as gerações detectadas no campo (Figura 1). Apesar de na safra agrícola 2002/2003 terem ocorrido cinco picos populacionais, observa-se que nas primeiras amostragens praticamente não foram encontrados insetos, diferentemente da safra agrícola anterior. Isso pode ser explicado pelo fato de que, no início do desenvolvimento dos frutos, a população do inseto é pequena, pois os frutos nesse estágio não são adequados para a sua multiplicação, com uma baixa viabilidade larval, quando comparada à cultivar Margarida, por exemplo. Além disso, outro fator que deve ser levado em conta é o fato de não ter sido observado se, no campo, ocorrem gerações sobrepostas. Assim, para implementação do modelo no campo, recomenda-se um acompanhamento local, para corrigir possíveis distorções, adaptando-o à ecologia do inseto.

A capacidade de postura de $S$. catenifer foi influenciada pela temperatura, uma vez que a $30^{\circ} \mathrm{C}$ as fêmeas colocaram 133,4 ovos, o que difere, estatisticamente, do número colocado nas temperaturas de $25^{\circ} \mathrm{C}$ e $20^{\circ} \mathrm{C}$, que foram de 318,3 e 318,9 ovos, respectivamente (Tabela 4). Para $S$. catenifer, diversos autores citaram diferentes valores de fecundidade, em temperaturas médias próximas a $25^{\circ} \mathrm{C}$. Hohmann \& Meneguim (1993) relataram capacidade média de postura de 164 ovos, enquanto Jaramillo et al. (1972) observaram posturas variáveis de 180 a 240 ovos, e Cervantes et al. (1999) relataram uma postura média de 206,3 ovos. Essa variação no número de ovos pode estar relacionada com as técnicas de criação utilizadas, bem como ao substrato utilizado para postura, e ao alimento utilizado para o desenvolvimento larval (polpa ou semente de abacate, diferentes variedades etc).

A temperatura também afetou a longevidade dos adultos de $S$. catenifer. Os machos viveram mais a $20^{\circ} \mathrm{C}$ (18,3 dias), tendo diferido, significativamente, da longevidade observada nas temperaturas de $25^{\circ} \mathrm{C}$

Tabela 3. Limiar térmico inferior de desenvolvimento (Tb), constante térmica (K), equação de regressão (1/D) e coeficiente de determinação $\left(\mathrm{R}^{2}\right)$ das fases de ovo, larval (lagarta), pupal (pupa) e ciclo biológico (ovo-adulto) de Stenoma catenifer, em condições de laboratório(1).

\begin{tabular}{lccccc}
\hline Fases/período & $\mathrm{Tb}\left({ }^{\circ} \mathrm{C}\right)(\mathrm{IC})$ & $\mathrm{K}(\mathrm{GD})$ & Equação de regressão & $\mathrm{R}^{2}$ & $\mathrm{P}$ \\
\hline Ovo & $9,14(8,76-10,13)$ & 82,3 & $1 / \mathrm{D}=0,0126 \mathrm{t}_{\mathrm{i}}-0,1111$ & 0,985 & $\leq 0,05$ \\
Lagarta & $8,26(7,44-9,51)$ & 398,4 & $1 / \mathrm{D}=0,0025 \mathrm{t}_{\mathrm{i}}-0,0207$ & 0,974 & $\leq 0,05$ \\
Pupa & $8,26(9,85-10,27)$ & 164,7 & $1 / \mathrm{D}=0,0061 \mathrm{t}_{\mathrm{i}}-0,0612$ & 0,995 & $\leq 0,05$ \\
Ovo-adulto & $8,89(8,61-9,34)$ & 644,5 & $1 / \mathrm{D}=0,0016 \mathrm{t}_{\mathrm{i}}-0,0138$ & 0,986 & $\leq 0,05$ \\
\hline
\end{tabular}

(1)IC: intervalo de confiança (95\%); GD: graus-dia. 


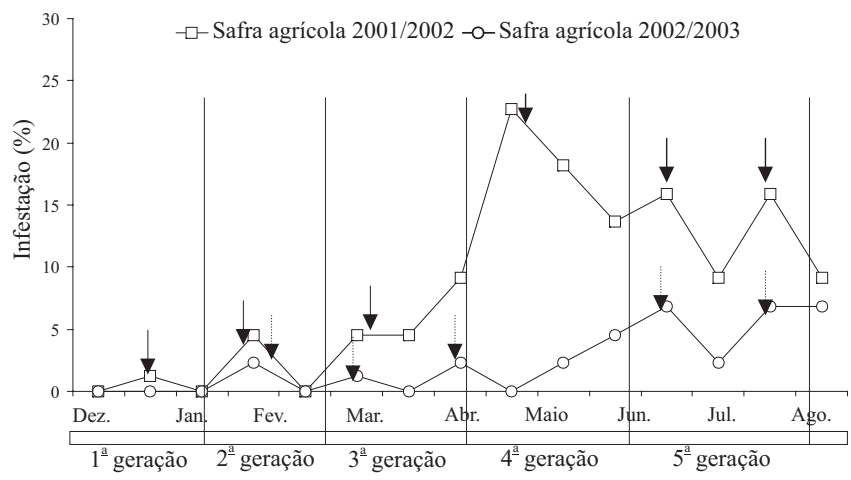

Figura 1. Flutuação populacional de Stenoma catenifer em abacate cultivar Breda, em São Tomás de Aquino, e provável número de gerações, com base na constante térmica do período ovo-adulto, determinada em laboratório. As prováveis gerações são representadas pelas linhas pontilhadas verticais. As setas contínuas e pontilhadas indicam os picos que podem corresponder às gerações, durante as safras agrícolas 2001/2002 e 2002/2003, respectivamente.

Tabela 4. Efeito de diferentes temperaturas $\left(20^{\circ} \mathrm{C}, 25^{\circ} \mathrm{C}\right.$ e $\left.30^{\circ} \mathrm{C}\right)$ sobre a fecundidade e a longevidade média ( \pm erro-padrão da média) de Stenoma catenifer, criado em sementes de abacate cultivar Breda, sob umidade relativa de $70 \pm 10 \%$ e fotófase de $14 \operatorname{horas}^{(1)}$.

\begin{tabular}{cccc}
\hline Temperatura & Número de & \multicolumn{2}{c}{ Longevidade (dias) } \\
\cline { 3 - 4 }$\left({ }^{\circ} \mathrm{C}\right)$ & ovos/fêmea & Macho & Fêmea \\
\hline 20 & $318,3 \pm 34,4 \mathrm{a}$ & $18,3 \pm 1,3 \mathrm{a}$ & $18,0 \pm 1,4 \mathrm{a}$ \\
25 & $318,9 \pm 28,5 \mathrm{a}$ & $13,4 \pm 1,2 \mathrm{~b}$ & $12,1 \pm 0,6 \mathrm{~b}$ \\
30 & $133,4 \pm 17,8 \mathrm{~b}$ & $10,6 \pm 0,6 \mathrm{~b}$ & $9,9 \pm 0,7 \mathrm{~b}$ \\
\hline $\mathrm{F}$ & 13,8919 & 15,8500 & 19,0198 \\
$\mathrm{P}$ & 0,00009 & 0,00004 & 0,00002 \\
$\mathrm{df}$ & 2 & 2 & 2 \\
$\mathrm{n}$ & 25 & 25 & 25 \\
\hline
\end{tabular}

(1)Médias seguidas da mesma letra, nas colunas, não diferem estatisticamente entre si, pelo teste de Tukey a 5\% de probabilidade.

(13,4 dias) e $30^{\circ} \mathrm{C}$ (10,6 dias) (Tabela 4). Para as fêmeas, a longevidade também diferiu estatisticamente, tendo sido maior a $20^{\circ} \mathrm{C}\left(18,0\right.$ dias) do que a $25^{\circ} \mathrm{C}$ (12,1 dias) e $30^{\circ} \mathrm{C}$ (9,9 dias) (Tabela 4). Pôde-se observar que a longevidade a $25^{\circ} \mathrm{C}$, tanto para machos quanto para fêmeas, foi bem superior aos 5 dias relatados por Acevedo et al. (1972), Boscán de Martínez \& Godoy (1982) e Hohmann \& Meneguim (1993).

\section{Conclusões}

1. A duração e a viabilidade, das diferentes fases do ciclo biológico de Stenoma catenifer são variáveis em função da temperatura.

2. O limiar térmico inferior de desenvolvimento, para as diferentes fases de $S$. catenifer, e para o período ovo-adulto é, exceto para pupas, inferior a $10^{\circ} \mathrm{C}$, e a constante térmica para o ciclo total é de 644,5 GD.

3. Com base nessas exigências térmicas, estimam-se 7,8 gerações da praga, durante o ano, e 5,1 gerações, durante o ciclo de produção.

4. A flutuação populacional de lagartas pode ser utilizada como um indicativo do número de gerações de S. catenifer, com base nas exigências térmicas da praga.

\section{Agradecimentos}

Ao Eng. Agrôn. José Gonçalves, Empresa Café Total, pelo financiamento da pesquisa e incentivo profissional; ao Eng. Agrôn. Peter Kasten Júnior, pela colaboração na pesquisa.

\section{Referências}

ACEVEDO, E.J.; VASQUEZ, J.T.; MOSS, C.S. Estudios sobre el barrenador del hueso y pulpa del aguacate Stenoma catenifer Walsingham (Lepidoptera: Stenomidae). Agrociencia, v.9, p.17-24, 1972.

ARELLANO CRUZ, G. El "barrenador del fruto del palto" Stenoma catenifer Wals. y su control natural en Chanchamayo y Satipo. Disponível em: <http://www.lamolina.edu.pe/ciencias/ ecologia/revista8.htm>. Acesso em: 8 fev. 2001.

ÁVILA, C.J.; MILANEZ, J.M.; PARRA, J.R.P. Previsão de ocorrência de Diabrotica speciosa utilizando-se o modelo de grausdia de laboratório. Pesquisa Agropecuária Brasileira, v.37, p.427432, 2002.

BLEICHER, E.; PARRA, J.R.P. Espécies de Trichogramma parasitóides de Alabama argilacea. III: Determinação das exigências térmicas de três populações. Pesquisa Agropecuária Brasileira, v.25, p.215-219, 1990.

BOSCÁN DE MARTÍNEZ, N.; GODOY, F.J. Apanteles sp. (Hymenoptera: Braconidae) parasito del taladrador del aguacate Stenoma catenifer Walsingham (Lepidoptera: Stenomidae) en Venezuela. Agronomía Tropical, v.32, p.319-321, 1982.

BOSCÁN DE MARTÍNEZ, N.; GODOY, F.J. Observaciones preliminares sobre la biología de Stenoma catenifer Walsingham (Lepidoptera: Stenomidae) taladrador del aguacate (Persea americana Mill.). Agronomía Tropical, v.34, p.205-208, 1984.

CERVANTES, P.L.; LYAL, C.H.C.; BROWN, V.K. The Stenomatine moth Stenoma catenifer Walsingham: a pre-dispersal seed predator of Greenheart (Chlorocardium rodiei (Schomb.) Rohwer, Richter \& Van der Werff) in Guyana. Journal of Natural History, v.33, p.531542, 1999.

COSTA LIMA, A.M. Insetos do Brasil: Lepidópteros. Rio de Janeiro: Escola Nacional de Agronomia, 1945. 379p. (Série didática, 7).

HADDAD, M.L.; PARRA, J.R.P.; MORAES, R.C.B. Métodos para estimar os limites térmicos inferior e superior de desenvolvimento de insetos. Piracicaba: Fealq, 1999. 29p. 
HIGLEY, L.G.; PEDIGO, L.P.; OSTLIE, K.R. DEGDAY: a program for calculating degree-days, and assumptions behind the degree-day approach. Environmental Entomology, v.15, p.999-1016, 1986.

HOHMANN, C.L.; MENEGUIM, A.M. Observações preliminares sobre a ocorrência da broca do abacate, Stenoma catenifer Wals. no Estado do Paraná. Anais da Sociedade Entomológica do Brasil, v.22, p.417-419, 1993.

HOHMANN, C.L.; SANTOS, W.J.; MENEGUIM, A.M. Avaliação de técnicas de manejo para o controle da broca-do-abacate, Stenoma catenifer (Wals.) (Lepidoptera: Oecophoridae). Revista Brasileira de Fruticultura, v.22, p.359-363, 2000.

HONÉK, A. Geographical variation in thermal requirements for insect development. European Journal of Entomology, v.93, p.303312, 1996.

JARAMILLO, E.A.; VÁSQUEZ, G; MOSS, C.S. Estudios sobre el barrenador del hueso y pulpa del aguacate Stenoma catenifer Walsingham (Lepidoptera: Stenomidae). Agrociencia, v.9, p.18-24, 1972.

MIHSFELDT, L.H. Biologia e exigências térmicas de Tuta absoluta (Meyrick, 1917) em dieta artificial. 1998. 87p. Tese
(Doutorado) - Escola Superior de Agricultura Luiz de Queiroz, Piracicaba.

NAVA, D.E. Comportamento de oviposição, bioecologia e subsídios para o controle biológico de Stenoma catenifer Walsingham, 1912 (Lepidoptera: Elachistidae). 2005. 140p. Tese (Doutorado) - Escola Superior de Agricultura Luiz de Queiroz, Piracicaba.

NAVA, D.E.; PARRA, J.R.P. Biology of Cerotoma arcuatus (Coleoptera: Chrysomelidae) and field validation of a laboratory model for temperature requirements. Journal of Economic Entomology, v.96, p.609-614, 2003.

PARRA, J.R.P. Biologia comparada de Perileucoptera coffeella (Guérin-Méneville, 1842) (Lepidoptera: Lyonetiidae) visando ao seu zoneamento ecológico no Estado de São Paulo. Revista Brasileira de Entomologia, v.29, p.45-76, 1985.

ZEISS, M.R.; KOEHLER, K.J.; PEDIGO, L.P. Degree-day requirements for development of the bean leaf beetle (Coleoptera: Chrysomelidae) under two rearing regimes. Journal of Economic Entomology, v.89, p.111-118, 1996. 